OPEN ACCESS

Edited by:

Luisa Lanfrancone,

European Institute of Oncology (IEO),

Italy

Reviewed by:

Chiara Fabbroni,

Istituto Nazionale dei Tumori (IRCCS),

Anna Caliò,

University of Verona, Italy

${ }^{*}$ Correspondence:

Lin Zhao

wz20010727@aliyun.com

${ }^{t}$ These authors have contributed equally to this work

Specialty section:

This article was submitted to Molecular and Cellular Oncology,

a section of the journal

Frontiers in Oncology

Received: 10 July 2020 Accepted: 26 October 2020 Published: 23 November 2020

Citation:

Xu J, Gong X-L, Wu H and Zhao L (2020) Case Report: Gastrointestinal PEComa With TFE3 Rearrangement Treated With Anti-VEGFR TKI Apatinib.

Front. Oncol. 10:582087. doi: 10.3389/fonc. 2020.582087

\section{Case Report: Gastrointestinal PEComa With TFE3 Rearrangement Treated With Anti-VEGFR TKI Apatinib}

\author{
Jiaming $\mathrm{Xu}^{1 \dagger}$, Xiao-Lei Gong ${ }^{1 \dagger}$, Huanwen $\mathrm{Wu}^{2}$ and Lin Zhao ${ }^{1 *}$ \\ ${ }_{1}^{1}$ Department of Medical Oncology, Peking Union Medical College Hospital, Chinese Academy of Medical Sciences and \\ Peking Union Medical College, Beijing, China, ${ }^{2}$ Department of Pathology, Peking Union Medical College Hospital, Chinese \\ Academy of Medical Sciences and Peking Union Medical College, Beijing, China
}

Perivascular epithelioid cell tumors (PEComas) are rare mesenchymal tumors. Unresectable malignant PEComas with TFE3 rearrangement have no recommended therapy to date. Here, we report the first case of malignant gastrointestinal PEComa with TFE3 rearrangement which has a response to the targeted therapy of an anti-VEGFR tyrosine kinase inhibitor (TKI), apatinib. A 31-year-old female was diagnosed with malignant gastrointestinal PEComa with TFE3 rearrangement and hepatic metastases. A resection of the giant retroperitoneal mass was performed. The patient received the antiVEGFR TKI apatinib to treat the hepatic metastasis. The tumor remained stable during apatinib treatment and the progression-free survival (PFS) lasted about 7 months. This case suggests that targeting the VEGFNEGFR signaling pathway may be an essential new therapeutic choice for TFE3-associated malignant PEComas.

Keywords: perivascular epithelioid cell tumors, TFE3, mammalian target of rapamycin inhibitor, anti-VEGFR, tyrosine kinase inhibitor, apatinib

\section{INTRODUCTION}

Perivascular epithelioid cell tumors (PEComas) are a group of rare mesenchymal tumors. They are composed of perivascular epithelioid cells (PECs) with distinctive histological and immunohistochemical characteristics (1). PECs are usually epithelioid or spindle-shaped, expressing both melanocytic and muscle markers (2). The PEComa family now includes angiomyolipoma (AML), clear cell "sugar" tumor (CCST) of the lung, lymphangioleiomyomatosis (LAM), and other neoplasms with aforementioned histological and immunohistochemical characteristics at various anatomical sites (3). AML and LAM are strongly associated with tuberous sclerosis complex (TSC), but another non-classic subset of PEComas associated with transcription factor E3 (TFE3) gene rearrangement has been reported in various sites of human body (2). Generally PEComas have a benign behavior but malignant cases have been reported at different sites (4). PEComas are usually treated with surgery (5). As an option of target therapy, mammalian target of rapamycin (mTOR) inhibitors have been proved safe and effective in the treatment of unresectable PEComas associated with TSC (6). However, mTOR inhibitors may lose effect in PEComas with TFE3 gene rearrangement (7), which calls for the investigation of more specific and effective therapies. Here we report a malignant case of PEComa in the gastrointestinal tract with hepatic 
metastasis and TFE3 gene rearrangement, which had a response to the treatment of the anti-VEGFR tyrosine kinase inhibitor (TKI), apatinib.

\section{CASE DESCRIPTION}

A 31-year-old woman presented with 3-month history of increased abdominal circumference and intermittent fever. The patient complained no other discomforts. She also denied any anomalies in the past and in the family history. In February 2018 the computed tomography (CT) scans showed a large mass in the upper left abdominal cavity. The mass measured about $16.8 \times 12.5 \mathrm{~cm}$, receiving blood supply from celiac trunk, splenic artery and branches of left gastric artery. Splenic vein drained blood flow of the tumor into portal vein. Other abnormalities include retroperitoneal lymphadenopathy, multiple hepatic metastases, enlarged left adrenal gland, and multiple small lymph nodes in the mesentery, pelvis and bilateral groin regions. The somatostatin receptor imaging and tomography showed increased expression of somatostatin receptor with necrosis in the left upper abdominal cavity and multiple hepatic space-occupying lesions.

A timeline of the episode of medical care is shown in Figure 1. A laparotomy and resection of the giant retroperitoneal mass (including partial posterior gastric wall, spleen, pancreatic body and tail, and partial left adrenal gland) was performed in March 2018. During surgery the mass was found located in the omental sac, closely associated with posterior gastric wall and was about $25 \times 25 \times 20 \mathrm{~cm}$. The mass disseminated widely in the abdominal cavity. Pathological findings revealed malignant PEComa of the gastrointestinal tract with diffuse expression of TFE3. Invasion of gastric mucosa and muscularis and pancreas with necrosis were noted. Lymph nodes showed chronic inflammation. Immunohistochemical stains (Figure 2) showed tumor cells positive for Melan-A (patchy), HMB-45 (strong and diffuse), vimentin (patchy) and TFE3 (modest and diffuse), and negative for PAX-8, S-100, Syn, CK7, desmin, Myo-D1, SMA, and calponin. Ki67 labeling index in the tumor cells was 30\%. RNAbased next-generation sequencing identified SFPQ-TFE3 fusion in tumor cells (Figure 3). A diagnosis of advanced malignant PEComa of the gastrointestinal tract with TFE3 rearrangement and hepatic metastases was made.

Unfortunately, the postoperative imaging showed progression of hepatic metastases (Figure 4A) and systemic treatment was needed. However, no effective therapy of PEComas with TFE3 expression was reported. Therefore, we decided to first use the mTOR inhibitor everolimus and see whether it worked on the patient. From May 6th she began to take mTOR inhibitor everolimus $10 \mathrm{mg}$ qd as first-line treatment for 3 months. Restaging CT showed tumor progression on everolimus treatment (Figure 4B). Considering the gastrointestinal origin of the hepatic metastases, from August $13^{\mathrm{th}}$ she was treated with $500 \mathrm{mg}$ qd apatinib, which is an anti-VEGFR2 TKI used in thirdline treatment of gastric cancer (GC) in China. The tumor remained stable (but with necrosis in the center, Figure 4C) and the progression-free survival (PFS) lasted about 7 months. During apatinib treatment the patient reported a remission of abdominal bloating. Mild proteinuria was confirmed through the routine urine test, and no other adverse events were observed. In March 2019, the hepatic metastases progressed (Figure 4D). In addition, multiple metastases of the tumor cells were detected in the right lung, greater omentum and pelvic cavity. Therefore we decided to try the combination therapy. From March $13^{\text {th }}$ 2019 the combination targeted therapy of everolimus $5 \mathrm{mg}$ qd and apatinib $250 \mathrm{mg}$ qd began, but no obvious improvement was observed. On June $4^{\text {th }}$ she stopped taking everolimus due to worsening cough, which might result from repeated pulmonary infections. From June 18th, she received anlotinib treatment for three courses with 2 weeks of medication and 1 week off. Unfortunately, the tumor progressed and the patient passed away in September 2019.

\section{DISCUSSION}

In 1992 Bonetti et al introduced the term "perivascular epithelioid cell (PEC)" to identify a novel cell type characterized by immunoreactivity with melanocyte markers, an epithelioid appearance, a clear or granular eosinophilic cytoplasm, and a perivascular distribution (8). In 1996 Zamboni et al. suggested the term PEComa for neoplasms totally composed of proliferation of PECs (9). The most reported cases of PEComas happened in the lung, liver and kidney. Gastrointestinal PEComas account for $20 \%$ to $25 \%$ of the rest reported cases in human body. The malignant rate of gastrointestinal PEComas is relatively high (up to 52\%), compared with those reported in other regions (for example, in the head and neck region the malignant rate is about 15.4\%) (10, 11). The patients may have various symptoms, including abdominal pain, obstruction, anemia, depending on tumor size and organs involved (10). In 2005, Folpe et al. (4) proposed the criteria for PEComas with malignant characteristics. Neoplasms are considered malignant with 2 or more following histologic features: size $>5 \mathrm{~cm}$, infiltrative, high nuclear grade and cellularity, mitotic rate $\geq 1 / 50 \mathrm{HPF}$, necrosis and vascular

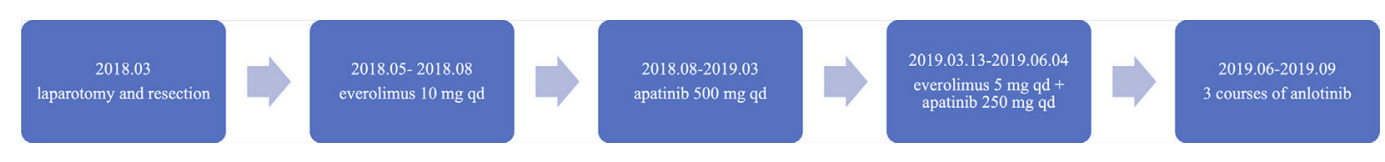

FIGURE 1 | Timeline with relevant data from the episode of medical care. 


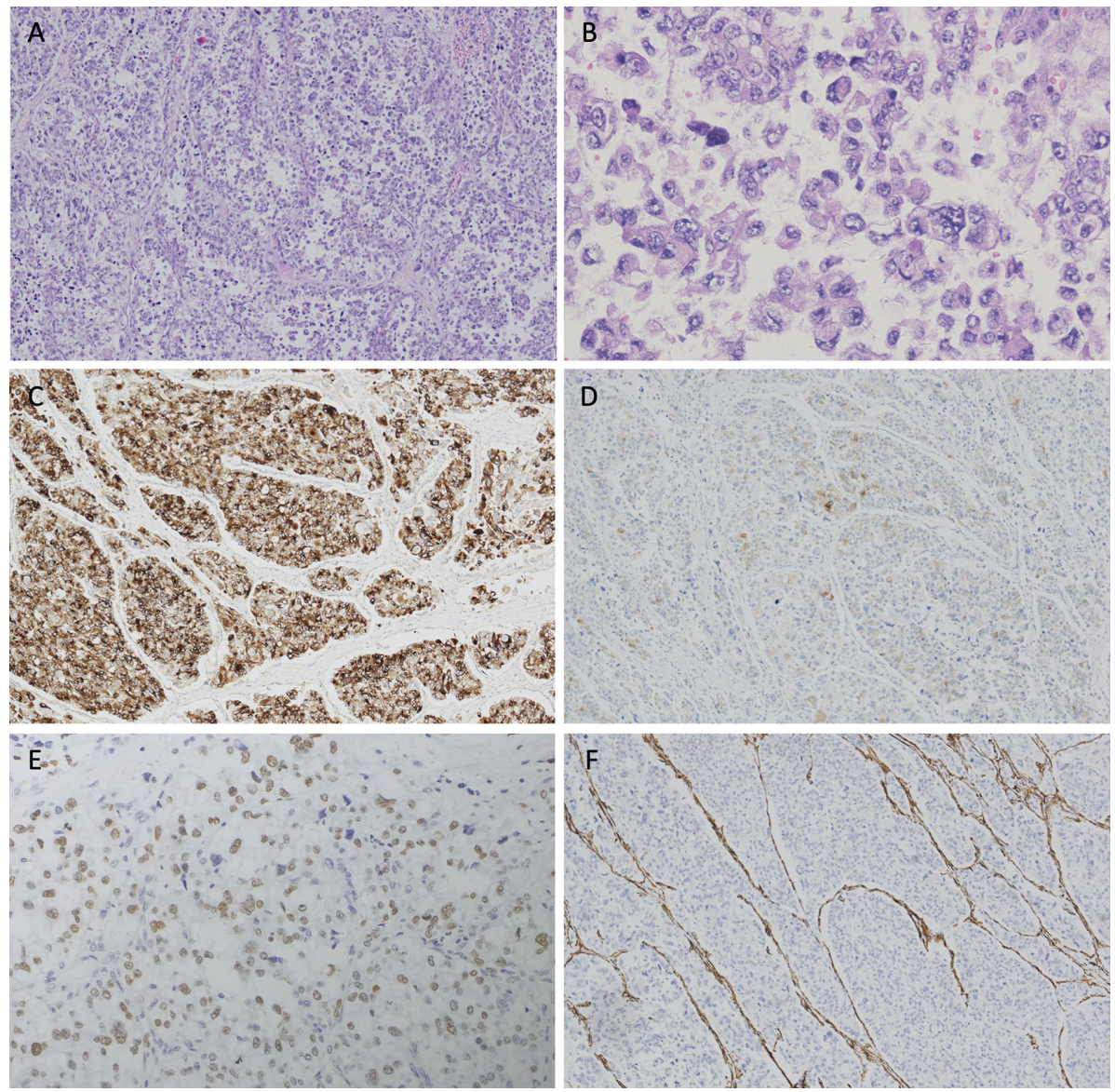

FIGURE 2 | Hematoxylin and eosin (H\&E) staining and immunohistochemical analyses of the resected tumor. (A) The tumor cells arrange in a diffuse pattern( $\times 4)$. (B) Epithelioid tumor cells are oval or polygonal, with clear or granular eosinophilic cytoplasm. Nucleoli are prominent in the vesicular nuclei. Increased mitotic activity and dysplasia are observed (×20). Tumor cells are positive for (C) HMB-45 (strong and diffuse), (D) Melan-A (patchy) and (E) TFE3 (modest and diffuse), and are negative for (F) SMA.

invasion. In our case the mass measured about $25 \times 25 \times 20 \mathrm{~cm}$, with infiltrative growth and necrosis in the gastric wall and pancreas as well as hepatic metastases. In addition, Ki67 labeling index in the tumor cells was $30 \%$. Therefore it was considered malignant.

PEComas express both melanocytic markers (such as HMB45, Melan-A, tyrosinase, and MiTF) and muscle markers (such as SMA, muscle myosin, and calponin) (2). Occasionally positive cases of desmin, cytokeratin, S-100, and CD1a are reported (2). The most sensitive melanocytic markers for the diagnosis of PEComas are HMB-45 and Melan-A (12). Our case demonstrated that tumors cell positive for HMB-45 and Melan-A, and negative for desmin, SMA, and S-100. Therefore, the diagnosis of PEComa was made.

Some PEComas are strongly associated with tuberous sclerosis complex (TSC) (2). The mutant genes in TSC are TSC1 and TSC2, whose gene products play a role in inhibiting mTOR. mTOR regulates cell division, therefore its hyperactivation contributes to TSC, resulting from deficiency of TSC1 and TSC2 (13). This mechanism indicates that mTOR inhibitor may serve as a therapy for TSC and TSC-related PEComas. Evidence has shown the efficacy of mTOR inhibitors, with partial or complete response in patients with TSC-related PEComas (6 of 11) (14). About 10\% of PEComas are associated with TFE3 rearrangement (4). These include tumors with SFPQ/PSF-TFE3 fusion or DVL2-TFE3 fusion (15), which are often observed in young patients without TSC2 alternations $(7,16)$. Different from the reported cases using FISH to detect the TFE3 rearrangement $(11,17)$, we conducted RNA-based next-generation sequencing technique to directly position the fusion locus, and found a SFPQ-TFE3 fusion in the tumor cells. A recent study suggests that PEComas with TFE3 rearrangement be reclassified as "melanotic Xp11 neoplasm" or "Xp11 neoplasm with melanocytic differentiation", because clinicopathologic data and outcome analysis indicate that these tumors have a closer relationship with alveolar soft part sarcomas (ASPS) instead of conventional PEComas with TSC mutations (18). Therefore, TFE3-rearranged PEComas may hypothetically be nonresponsive to mTOR inhibitor therapy (19). Our patients received mTOR inhibitor everolimus. However, the first-line treatment showed no response to everolimus and tumor 


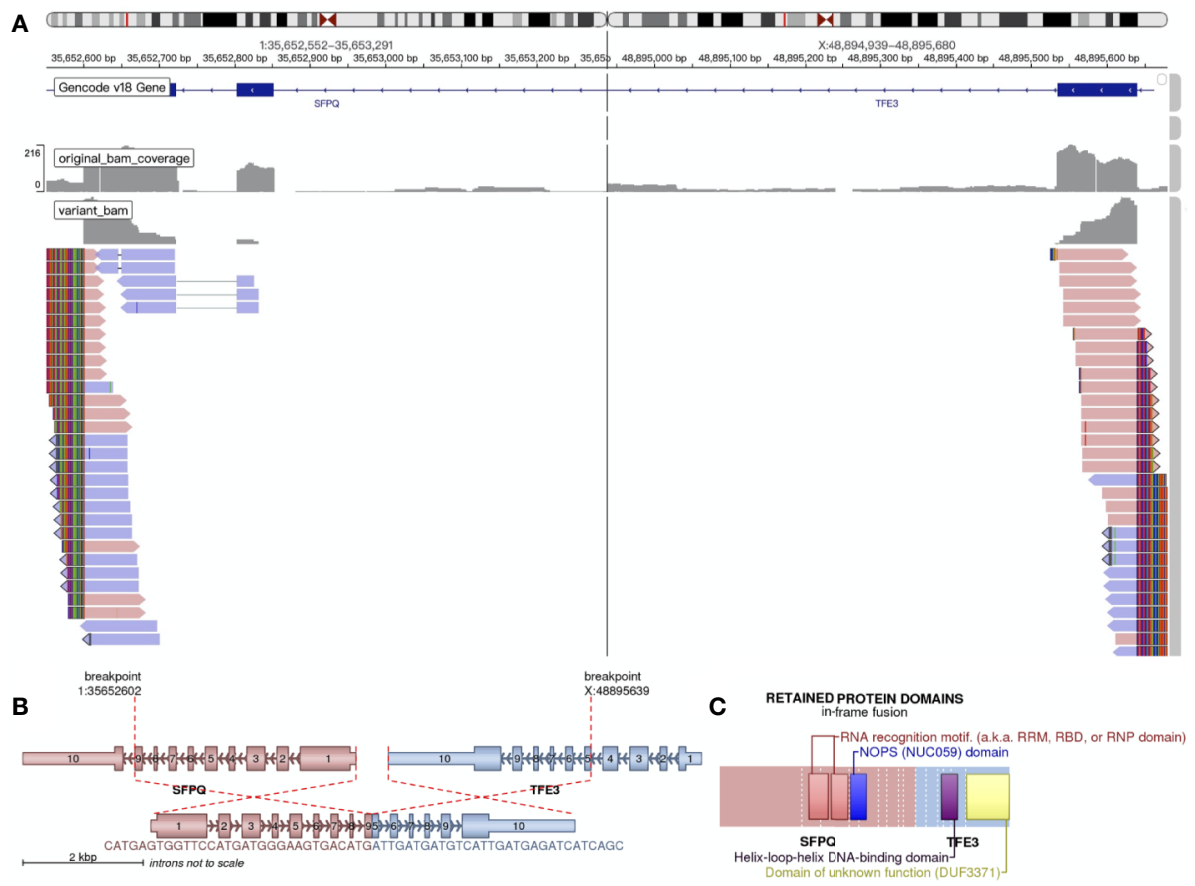

FIGURE 3 | RNA-based next-generation sequencing identified SFPQ-TFE3 fusion in tumor cells. (A) Visualization of the sequencing results through the Integrative Genomics Viewer (IGV). A SFPQ-TFE3 fusion was showed. (B) shows the location where the fusion took place. (C) shows the retained protein domains of the fusion protein.

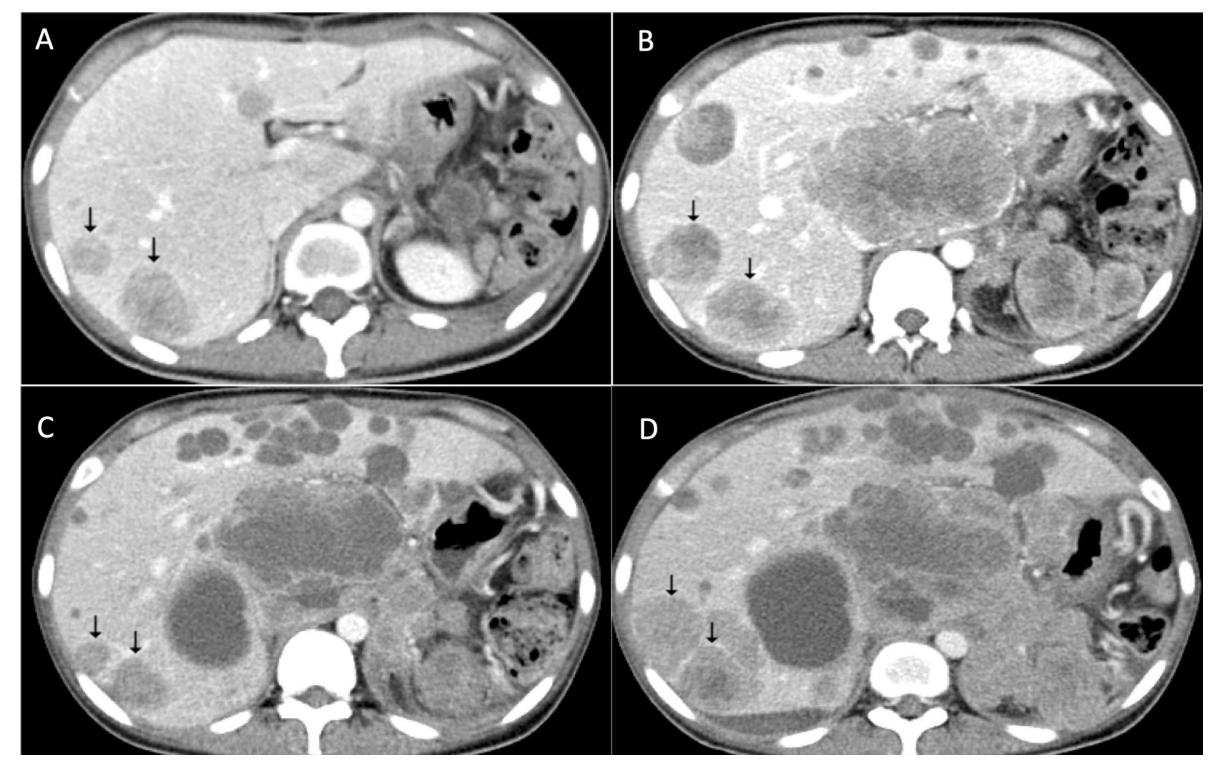

FIGURE 4 | Response of apatinib for metastatic PEComa of the liver. (A) Multiple hepatic metastases revealed after surgery. (B) Tumor progression after everolimus treatment. (C) Response of hepatic metastases after apatinib treatment. (D) Tumor progression in April 2019.

progression, which appeared to support the inefficacy of mTOR inhibitors in PEComas with TFE3 rearrangement.

The inefficacy of mTOR inhibitors in our case urged us to find a better therapy. Here we turned to VEGFR inhibitors. It was reported that a rise in serum VEGF-D concentration is often observed in PEComa patients, which is associated with clinical presentation of PEComa (20), suggesting that targeting the VEGF/VEGFR signaling pathway should be a potential 
treatment for malignant PEComas. An attenuation of VEGF-D upregulation in serum and inhibition of LAM is observed in a mouse model treated with VEGFR inhibitor axitinib (21). In our case we are the first to report the therapy of VEGFR-2 inhibitor apatinib to treat PEComa with TFE3 rearrangement. Apatinib is a newly developed anti-angiogenetic agent in China used in third-line treatment of GC, which is an anti-VEGFR2 TKI and has shown efficacy in GC, hepatocellular carcinoma (HCC), and non-small cell lung cancer (NSCLC) (22). The anti-angiogenetic effect of apatinib may help to normalize tumor angiogenesis and to enhance the sensitivity of chemotherapy (23). After receiving apatinib treatment the tumor remained stable for 7 months without progression, which showed that the anti-VGEFR effect might be useful in the treatment of malignant PEComas. Generally, the adverse reactions of apatinib treatment are associated with hypertension, urine protein, hand foot syndrome and gastrointestinal reaction (diarrhea) (22). In our case, mild proteinuria was confirmed in the patient, while hypertension was not observed.

When the tumor finally progressed, we chose the combination therapy of the anti-VEGFR TKI apatinib and an mTOR inhibitor everolimus. However, the outcome was not satisfactory and the tumor kept on progressing. By contrast, a recent study reported that a patient with malignant uterine PEComa receiving combination targeted therapy of a VEGFR inhibitor (sorafenib) with an mTOR inhibitor (sirolimus) has shown a complete response (24). Therefore, whether the combination targeted therapy is useful in TFE3-associated malignant PEComas needs more investigation.

Recently the largest international retrospective series analyzed the therapeutic effects of VEGFR inhibitors (pazopanib, sorafenib, and sunitinib) in advanced PEComas. In the retrospective series, the ORR for VEGFR inhibitors was $8.3 \%$, and the median PFS was 5.4 months (25). However, the limitation of the reported VEGFR inhibitors therapy was that the authors did not mention the mutational status of the patients. In other words, we still don't know whether the therapeutic effects of anti-VEGFR TKI are different between TSC1/2mutated and TFE3-associated PEComas. By contrast, our case specifically shows the therapeutic potential of anti-VEGFR TKI in TFE3-associated PEComas. We consider that it's an innovative perspective.

Another recent retrospective series reported the reversion of the resistance to mTOR inhibitors by adding exemestane in advanced PEComas (with a median PFS of 7 months and a median DOR of 11 months) (26), which indicated that the combination of mTOR inhibitors and exemestane would be effective after progression on mTOR inhibitor monotherapy. However, the combination therapy of mTOR inhibitors and exemestane were effective only in TSC1/2-mutated PEComas, whether it has effect in TFE3-associated PEComas still needs more exploration. By contrast, in this case report the antiVEGFR TKI apatinib directly took effect in advanced PEComas with TFE3 rearrangement, which suggested a better therapy for TFE3-associated PEComas than the combination of mTOR inhibitors and exemestane.
Through our exploration, we consider that the anti-VEGFR effect may play a more important role in the treatment of PEComa with TFE3 rearrangement. Therefore, targeting the VEGF/VEGFR signaling pathway may be a useful strategy for unresectable PEComas, especially those with TFE3 rearrangement and a limited response to mTOR inhibitors, but to what extent the tumor can be restrained needs more investigation. Future studies should explore the mechanism of apatinib inducing the clinical effects, and whether the combination therapy is useful in TFE3associated malignant PEComas.

\section{CONCLUSIONS}

For malignant PEComas with TFE3 rearrangement, the mTOR inhibitor therapy shows limited effect, calling for new therapies. One of the strategies is to target another VEGF/VEGFR signaling pathway. Here we are the first to report that VEGFR inhibitor apatinib had a 7 months duration of response in a patient with advanced malignant PEComa with TFE3 rearrangement. This case suggests that targeting the VEGF/VEGFR signaling pathway may be an essential new therapeutic choice for TFE3-associated malignant PEComas.

\section{DATA AVAILABILITY STATEMENT}

The original contributions presented in the study are included in the article/supplementary material. Further inquiries can be directed to the corresponding author.

\section{ETHICS STATEMENT}

Informed consent was obtained from the patient for publication of this case report and any accompanying images. The patient gave consent to publish this case report, and read the article and confirmed its content.

\section{AUTHOR CONTRIBUTIONS}

JX, X-LG and LZ designed the study, conducted the literature review, collected the data and wrote and revised the draft manuscript and subsequent manuscripts. HW conducted the experiments. JX and X-LG contributed equally to the manuscript. All authors contributed to the article and approved the submitted version.

\section{ACKNOWLEDGMENTS}

I want to give my sincere gratitude to Tingting You and Junjie $\mathrm{Gu}$ who helped me collect the needed data when I was trapped at home and had no access to the information I needed. Also I am grateful to all the authors for these amazing jobs and insightful suggestions. 


\section{REFERENCES}

1. Martignoni G, Pea M, Reghellin D, Zamboni G, Bonetti F. PEComas: the past, the present and the future. Virchows Arch (2008) 452(2):119-32. doi: 10.1007/ s00428-007-0509-1

2. Thway K, Fisher C. PEComa: morphology and genetics of a complex tumor family. Ann Diagn Pathol (2015) 19(5):359-68. doi: 10.1016/ j.anndiagpath.2015.06.003

3. Flope AL, Kwiatkowski DJ. Perivascular epithelioid cell neoplasms: pathology and pathogenesis. Hum Pathol (2010) 41(1):1-15. doi: 10.1016/ j.humpath.2009.05.011

4. Flope AL, Mentzel T, Lehr H-A, Fisher C, Balzer BL, Weiss SW. Perivascular epithelioid cell neoplasms of soft tissue and gynecologic origin: a clinicopathologic study of 26 cases and review of the literature. Am J Surg Pathol (2005) 29(12):1558-75. doi: 10.1097/01.pas.0000173232.22117.37

5. Bleeker JS, Quevedo JF, Folpe AL. "Malignant” perivascular epithelioid cell neoplasm: risk stratification and treatment strategies. Sarcoma (2012) 541626 (10):26. doi: 10.1155/2012/541626

6. Bissler JJ, Kingswood JC, Radzikowska E, Zonnenberg BA, Frost M, Belousova E, et al. Everolimus for renal angiomyolipoma in patients with tuberous sclerosis complex or sporadic lymphangioleiomyomatosis: extension of a randomized controlled trial. Nephrol Dial Transplant (2016) 31(1):111-9. doi: $10.1093 / \mathrm{ndt} / \mathrm{gfv} 249$

7. Malinowska I, Kwiatkowski DJ, Weiss S, Martignoni G, Netto G, Argani P. Perivascular epithelioid cell tumors (PEComas) harboring TFE3 gene rearrangements lack the TSC2 alterations characteristic of conventional PEComas: further evidence for a biological distinction. Am J Surg Pathol (2012) 36(5):783-4. doi: 10.1097/PAS.0b013e31824a8a37

8. Bonetti F, Pea M, Martignoni G, Zamboni G. PEC and sugar. Am J Surg Pathol (1992) 16(3):307-8. doi: 10.1097/00000478-199203000-00013

9. Zamboni G, Pea M, Martignoni G, Martignoni G, Zancanaro C, Faccioli G, et al. Clear cell "sugar" tumor of the pancreas. A novel member of the family of lesions characterized by the presence of perivascular epithelioid cells. Am J Surg Pathol (1996) 20(6):722-30. doi: 10.1097/00000478-199606000-00010

10. Chen Z, Han S, Wu J, Xiong M, Huang Y, Chen J, et al. A systematic review: perivascular epithelioid cell tumor of gastrointestinal tract. Medicine (2016) 95 (28):e3890. doi: 10.1097/MD.0000000000003890

11. Saluja K, Thomas J, Zhang S, Sturgis EM, Jain KS, Prieto VG, et al. Malignant perivascular epithelioid cell tumor of the oropharynx with strong TFE3 expression mimicking alveolar soft part sarcoma: a case report and review of the literature. Hum Pathol (2018) 76:149-55. doi: 10.1016/j.humpath.2017.11.016

12. Chang KL, Flope AL. Diagnostic utility of microphthalmia transcription factor in malignant melanoma and other tumors. Adv Anat Pathol (2001) 8 (5):273-5. doi: 10.1097/00125480-200109000-00004

13. Franz DN, Krueger DA. mTOR inhibitor therapy as a disease modifying therapy for tuberous sclerosis complex. Am J Med Genet C Semin Med Genet (2018) 178(3):365-73. doi: 10.1002/ajmg.c.31655

14. Dickson MA, Schwartz GK, Antonescu CR, Kwiatkowski DJ, Malinowska IA. Extrarenal perivascular epithelioid cell tumors (PEComas) respond to mTOR inhibition: clinical and molecular correlates. Int J Cancer (2013) 132(7):17117. doi: 10.1002/ijc. 27800

15. Agaram NP, Sung YS, Zhang L, Chen CL, Chen HW, Singer S, et al. Dichotomy of Genetic Abnormalities in PEComas With Therapeutic Implications. Am J Surg Pathol (2015) 39(6):813-25. doi: 10.1097/PAS.0000000000000389
16. Argani P, Aulmann S, Illei PB, Netto GJ, Ro J, Cho HY, et al. A distinctive subset of PEComas harbors TFE3 gene fusions. Am J Surg Pathol (2010) 34 (10):1395-406. doi: 10.1097/PAS.0b013e3181f17ac0

17. Shon W, Kim J, Sukov W, Reith J. Malignant TFE3-rearranged perivascular epithelioid cell neoplasm (PEComa) presenting as a subcutaneous mass. $\mathrm{Br} J$ Dermatol (2016) 174(3):617-20. doi: 10.1111/bjd.14173

18. Wang XT, Fang R, Zhang RS, Ye SB, Li R, Wang X, et al. Malignant melanotic $\mathrm{Xp11}$ neoplasms exhibit a clinicopathological spectrum and gene expression profiling akin to alveolar soft part sarcoma: a proposal for reclassification. J Pathol (2020) 251(4):365-77. doi: 10.1002/path.5470

19. Schoolmeester JK, Dao LN, Sukov WR, Wang L, Park KJ, Murali R, et al. TFE3 translocation-associated perivascular epithelioid cell neoplasm (PEComa) of the gynecologic tract: morphology, immunophenotype, differential diagnosis. Am J Surg Pathol (2015) 39(3):394-404. doi: 10.1097/PAS.0000000000000349

20. Radzikowska E, Paulina J, Sobiecka M, Chorostowska-Wynimko J, Wiatr E, Kuś J, et al. Correlation of serum vascular endothelial growth factor-D concentration with clinical presentation and course of lymphangioleiomyomatosis. Respir Med (2015) 109(11):1469-75. doi: 10.1016/j.rmed.2015.09.005

21. Atochina-Vasserman EN, Abramova E, James ML, Rue R, Liu AY, Ersumo NT, et al. Pharmacological targeting of VEGFR signaling with axitinib inhibits Tsc2-null lesion growth in the mouse model of lymphangioleiomyomatosis. Am J Physiol Lung Cell Mol Physiol (2015) 309(12):2. doi: 10.1152/ ajplung.00262.2015

22. Xue JM, Astère M, Zhong MX, Lin H, Shen J, Zhu YX. Efficacy and safety of apatinib treatment for gastric cancer, hepatocellular carcinoma and non-small cell lung cancer: a meta-analysis. Onco Targets Ther (2018) 11:6119-28. doi: 10.2147/OTT.S172717

23. Lin MI, Sessa WC. Antiangiogenic therapy: creating a unique "window" of opportunity. Cancer Cell (2004) 6(6):529-31. doi: 10.1016/S1535-6108(04) 00340-X

24. Gao F, Huang C, Zhang Y, Sun R, Zhang Y, Wang H, et al. Combination targeted therapy of VEGFR inhibitor, sorafenib, with an mTOR inhibitor, sirolimus induced a remakable response of rapid progressive Uterine PEComa. Cancer Biol Ther (2016) 17(6):595-8. doi: 10.1080/ 15384047.2016.1167290

25. Sanfilippo R, Jones RL, Blay JY, Le Cesne A, Provenzano S, Antoniou G, et al. Role of Chemotherapy, VEGFR Inhibitors, and mTOR Inhibitors in Advanced Perivascular Epithelioid Cell Tumors (PEComas). Clin Cancer Res (2019) 25(17):5295-300. doi: 10.1158/1078-0432.CCR-19-0288

26. Sanfilippo R, Fabbroni C, Fuca G, Fumagalli E, Morosi C, Sbaraglia M, et al. Addition of Antiestrogen Treatment in Patients with Malignant PEComa Progressing to mTOR Inhibitors. Clin Cancer Res (2020) 26(20):5534-8. doi: 10.1158/1078-0432.CCR-20-1191

Conflict of Interest: The authors declare that the research was conducted in the absence of any commercial or financial relationships that could be construed as a potential conflict of interest.

Copyright (c) $2020 \mathrm{Xu}$, Gong, Wu and Zhao. This is an open-access article distributed under the terms of the Creative Commons Attribution License (CC BY). The use, distribution or reproduction in other forums is permitted, provided the original author(s) and the copyright owner(s) are credited and that the original publication in this journal is cited, in accordance with accepted academic practice. No use, distribution or reproduction is permitted which does not comply with these terms. 